EPJ Web of Conferences 86,00063 (2015)

DOI: $10.1051 /$ epjconf/ 20158600063

(C) Owned by the authors, published by EDP Sciences, 2015

\title{
How signatures of quasifission evolve in reactions forming Curium
}

\author{
E. Williams ${ }^{1, a}$, D. J. Hinde ${ }^{1}$, M. Dasgupta ${ }^{1}$, R. du Rietz ${ }^{1, b}$, I. P. Carter ${ }^{1}$, M. Evers ${ }^{1}$, D. H. Luong ${ }^{1}$, S. D. McNeil ${ }^{1}$, D. \\ C. Rafferty ${ }^{1}$, K. Ramachandran ${ }^{1, c}$, and A. Wakhle ${ }^{1}$ \\ ${ }^{1}$ Department of Nuclear Physics, Research School of Physical Sciences and Engineering, The Australian National University, \\ Canberra, ACT 0200, Australia
}

\begin{abstract}
Quasifission, a fission-like reaction outcome in which no compound nucleus forms, is an important competitor to fusion in reactions used for super-heavy element formation. The precise mechanisms driving the competition between quasifission and fusion are poorly understood. To explore the influence reaction parameters have on quasifission probabilities, an investigation into the evolution of quasifission signatures as a function of entrance channel parameters is required. Using the Australian National University's 14UD tandem accelerator and CUBE detector for two-body fission studies, measurements were made for a diverse range of reactions forming isotopes of Curium. Observables known to reveal signs of quasifission-namely mass ratio spectra, mass-angle distributions, and angular anisotropies-were extracted. Evidence of quasifission was observed in all reactions, but the observables showing evidence of quasifission were not the same for all reactions. A link between this evolution and reaction timescales will be discussed.
\end{abstract}

\section{Introduction}

A reaction outcome known as quasifission is one of the most important inhibitors of fusion in reactions leading to heavy and superheavy element formation. In quasifission, the di-nuclear system formed following capture begins the equilibration process, but splits apart again before forming the compact equilibrium shape required in fusion. For reactions leading to quasifission, no compound nucleus forms; as such, quasifission is a direct competitor to fusion. Because of this competition between fusion and quasifission, the latter has long been the subject of intense study (see, e.g., Refs. [1-19]).

The role quasifission plays in suppressing compound nucleus formation has several consequences. In reactions dominated by quasifission, reaction times are faster than those dominated by fusion fission by as much as four orders of magnitude $[4,16]$. Such reactions have been shown to be strongly influenced by entrance channel variables $[4,17,20,21]$, and have been associated with a reduction in evaporation residue cross sections [11]. To date, an understanding of how signatures of quasifission evolve with reaction timescales has not been achieved.

Quasifission presents a considerable experimental challenge because individual fission events resulting from this process have identical characteristics to those observed in fusion-fission. But in quasifission, the elongated projectile-target system never reaches equilibrium in the case of quasifission. Instead, it sticks together just long

\footnotetext{
ae-mail: elizabeth.williams@anu.edu.au

${ }^{b}$ Current address: Malmö University, Malmö 205 06, Sweden

${ }^{c}$ Permanent address: Nuclear Physics Division, Bhabha Atomic Research Centre, Mumbai 400085, India
}

enough for some mass-equilibration to occur. In reactions dominated by quasifission, the distributions of fission fragment characteristics should bear some signatures of this dynamic collision process.

Following Ref. [18], we will focus on three signatures of quasifission: mass distributions, angular anisotropies, and mass-angle distributions. Mass distributions have been shown to exhibit enhanced widths in reactions dominated by quasifission in comparison to equivalent reactions dominated by fusion-fission [13, 22]. Angular anisotropies that show enhancement relative to transition state model predictions have also been used to identify reactions in which quasifission is a possible outcome $[2,6,23]$. Finally, mass-angle distributions (MADs) [4, 16, 19] have been shown to provide a clear signature of quasifission in reactions occurring primarily over relatively short timescales. While all these observables are related, each is sensitive to different aspects of the quasifission process. As such, a careful study of these observables in reactions leading to Curium will allow us to examine the evolution of quasifission as a function of entrance channel parameters. In particular, a relationship between these signatures and quasifission timescales will be explored.

\section{Methods}

\subsection{Experiment}

To observe how entrance channel parameters influence quasifission signatures, several experiments were performed using the Australian National University's 14UD tandem accelerator, and, where necessary, the ANU superconducting linear accelerator. Pulsed beams ranging from 


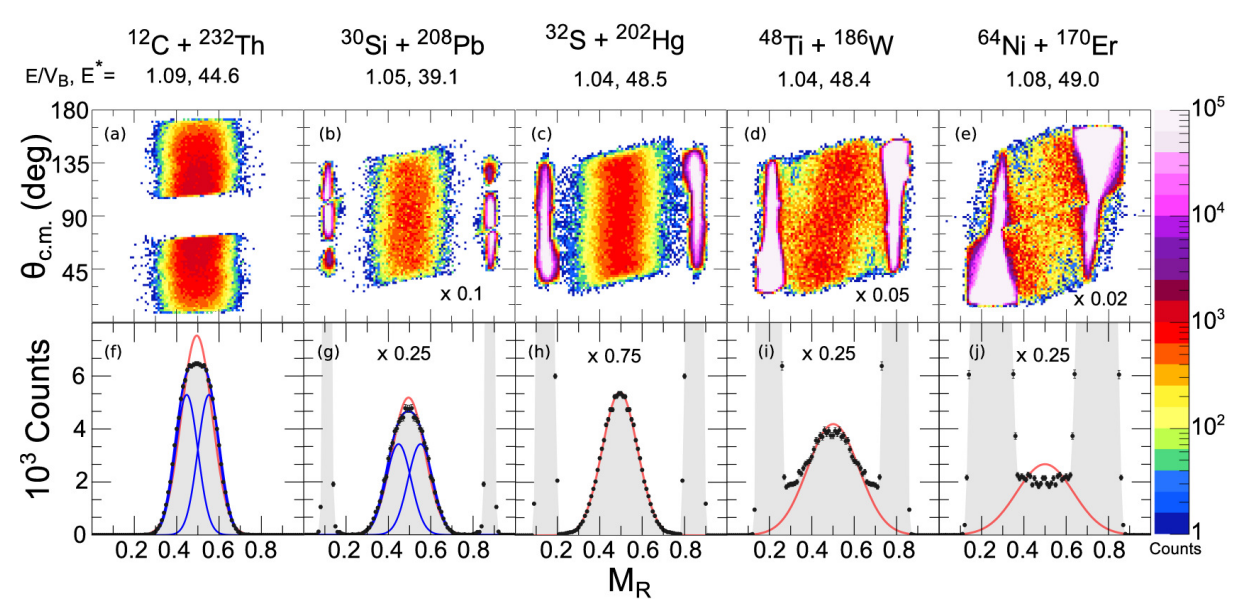

Figure 1. (a-e) MADs for all five reactions studied in this work. (f-j) Mass ratios for each studied reaction, compared with a single (red) or, where applicable, a dual (blue) Gaussian fit, as described in Ref. [18]. For all results in this figure, only one energy for each reaction is shown for brevity; similar results were obtained for other excitation energies.

${ }^{12} \mathrm{C}$ to ${ }^{64} \mathrm{Ni}$, of width $\sim 1 \mathrm{~ns}$ and separation $\sim 106 \mathrm{~ns}$, bombarded targets ranging from ${ }^{232} \mathrm{Th}$ to ${ }^{170} \mathrm{Er}$. All reactions formed isotopes of Curium. A summary of the reactions chosen and any relevant reaction parameters is provided in Table I of Ref. [18].

The ANU CUBE detector system [8] was used to observe fission fragments originating from each reaction. The CUBE detector consists of two position-sensitive Multi-Wire Proportional Counters (MWPCs) offering a large solid angle coverage. To make absolute cross section measurements possible, Si monitor detectors were placed at forward angles in symmetric locations about the beam axis. Depending on the reaction kinematics and required angular coverage, two different CUBE configurations (shown in Fig. 1 of Ref. [18]), were used.

For each experiment, mass ratios $\left(M_{R} \equiv M 1 /(M 1+\right.$ $M 2)$ ), MADs, and, where necessary, angular anisotropies, were obtained. For a full explanation of the kinematic reconstruction, event classification, and cross section normalization methods used in this work, see Ref. [18].

\subsection{Theory}

Because quasifission and fusion-fission outcomes are typically possible in the same reactions and cannot be separated by experimental means, all three of the observables used in this work necessarily represent the sum of both quasifission and fusion-fission distributions. In the case of angular anisotropies, in particular, this means there is no clear and model-independent signature of the presence of quasifission. Instead, transition state model calculations that assume that compound nucleus formation precedes fission serve to benchmark fusion-fission angular anisotropies; significant deviations from these predictions are taken as evidence of the presence of quasifission.

In the current work, angular anisotropy calculations were based on the code JOANNE2 [24]. For these calculations, we used the transition state model (TSM) [25] as described in Ref. [26], as well as partial wave distributions ( $\ell$-distributions) following fusion obtained using a

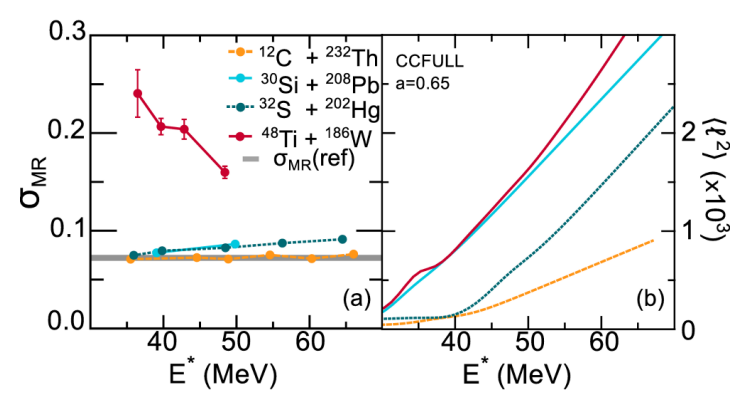

Figure 2. (a) Mass widths extracted for reactions using the lightest four projectiles, plotted with respect to excitation energy $E^{*}$ in units of MeV. (b) $\left\langle\ell^{2}\right\rangle$ values calculated using CCFULL for a diffuseness $a=0.65$ for the four reactions shown in (a).

variant of the coupled-channels model CCFULL [27, 28]. A detailed discussion of the theoretical calculations used in this work can be found in Ref. [18].

\section{Results}

Mass ratios and MADs were measured for all reactions studied in this work. Angular anisotropies were only studied for those reactions in which no clear evidence of quasifission was found in these first two observables.

\subsection{Mass ratios}

Mass ratios for all full momentum transfer events in each reaction were extracted; a sample mass ratio for each reaction is shown the bottom row of in Fig. 1. With the exception of results from ${ }^{64} \mathrm{Ni}+{ }^{170} \mathrm{Er}$, in which the the mass ratios exhibited a minimum at $M_{R}=0.5$, each obtained mass ratio was fit with a single Gaussian function. The width obtained from this fit is shown as a function of excitation energy $E^{*}$ in Fig. 2 (a). The average width for the reaction ${ }^{12} \mathrm{C}+{ }^{232} \mathrm{Th}$, in which quasifission is not a likely outcome, is used as a reference width $\left(\sigma_{M R}(\right.$ ref $\left.)\right)$. As one can 

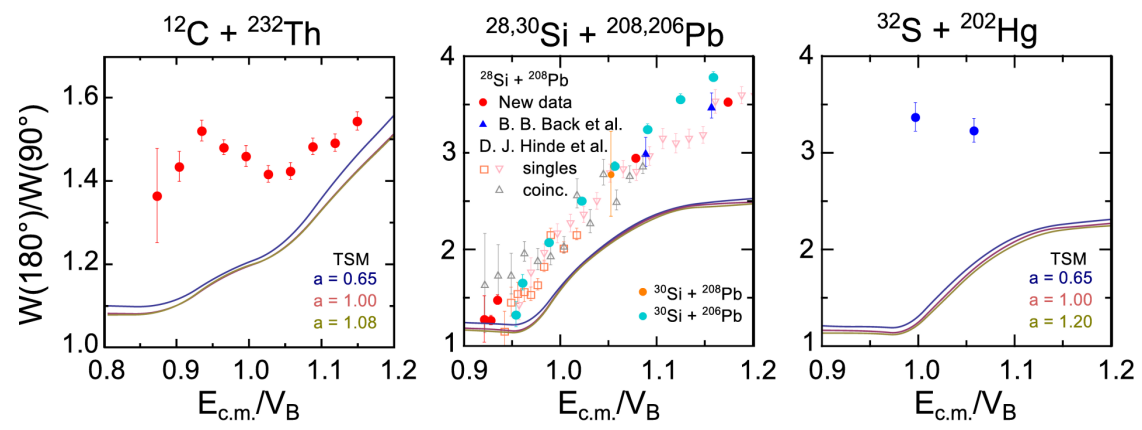

Figure 3. Angular anisotropies extracted for reactions using the lightest four projectiles, plotted with respect to excitation energy $E^{*}$ in units of MeV. The angular anisotropy data for ${ }^{12} \mathrm{C}+{ }^{232} \mathrm{Th}$ are from Ref. [29]; data for ${ }^{28} \mathrm{Si}+{ }^{208} \mathrm{~Pb}$ are from Refs. [30] and [7]. All other data are from this work.

see from this plot, all reactions using projectiles higher than ${ }^{12} \mathrm{C}$ exhibited wider mass distributions than $\sigma_{M R}(\mathrm{ref})$ for most or all excitation energies. The standout case of ${ }^{48} \mathrm{Ti}+{ }^{186} \mathrm{~W}$ will be discussed below.

These differences alone are not sufficient to conclude that quasifission is an important reaction outcome for all these reactions; angular momentum does play a role in determining mass distributions. However, a sample CCFULL calculation of $\left\langle\ell^{2}\right\rangle$, where $\left\langle L^{2}\right\rangle \equiv\left\langle\ell^{2}\right\rangle \hbar^{2}$, is shown in Fig. 2 (b). From this, one can see that $\left\langle\ell^{2}\right\rangle$ values for ${ }^{32} \mathrm{~S}+{ }^{202} \mathrm{Hg}$ are very similar to those for ${ }^{12} \mathrm{C}+{ }^{232} \mathrm{Th}$ below $E^{*} \sim 40 \mathrm{MeV}$; nevertheless, mass widths for the former are measurably larger than the reference width. This suggests that quasifission may be important for ${ }^{32} \mathrm{~S}+{ }^{202} \mathrm{Hg}$.

\subsection{Mass-angle distributions}

MADs for all reactions are shown for one excitation energy in Fig. 1 (a-e). For reactions with the lightest three projectiles, Fig. 1 (a-c), no correlation between mass and angle is evident in the MADs. For reactions with the heaviest two projectiles, Fig. 1 (d-e), however, a mass-angle correlation is evident, and is most extreme for ${ }^{64} \mathrm{Ni}+{ }^{170} \mathrm{Er}$. The correlation is thought to come about due to the short reaction timescales present in relatively fast quasifission processes, as described in Refs. [4, 16]. It is this correlation that primarily leads to the dramatically different mass ratios observed in ${ }^{48} \mathrm{Ti}+{ }^{186} \mathrm{~W}$ and ${ }^{64} \mathrm{Ni}+{ }^{170} \mathrm{Er}$, Fig. $1(\mathrm{i}-\mathrm{j})$.

\subsection{Angular anisotropies}

For the three reactions involving the lightest projectiles, mass widths and MADs did not offer conclusive evidence of the presence of quasifission. This does not mean that quasifission is not a possible outcome; MADs in particular will only offer evidence of quasifission in the form of a mass-angle correlation for sufficiently short $\left(<10^{-20}\right.$ s) reaction timescales, corresponding to relatively fast quasifission processes. To determine how well each reaction conformed with traditional fusion-fission, angular anisotropies were observed and compared to transition state model calculations.
The results of these comparisons are shown in Fig. 3, plotted as functions of $E_{c . m} / V_{B}$. To account for the uncertainty in the Woods-Saxon potential diffuseness parameter (a) used in the $\ell$-distribution calculations provided as inputs to the model, three different transition state model calculations are shown for each reaction. Even large changes in the diffuseness parameter have only a small influence on the calculated angular anisotropies. In Fig. 3, transition state model calculations are plotted as solid lines, while data points are distinct symbols with error bars. For any of the three reactions shown in Fig. 3, measurements do not agree with the model calculations. For all three cases, then, angular anisotropies offer evidence that quasifission is a possible outcome.

\section{Discussion and Conclusions}

The above results have shown that all five reactions studied indicated some evidence of quasifission in at least one of the fission fragment observables. While we cannot separate out fusion-fission and quasifission event-by-event, nor can we robustly determine how significant each process is in each reaction, we can say this: quasifission is clearly an important reaction outcome for all reactions studied here, including those with the lightest projectile.

The clearest (and most model-independent) indicator of quasifission was found in the MADs. In the reactions involving the heaviest projectiles, quasifission occurring on short time scales (less than a single rotation after capture) can be identified from the presence of a mass-angle correlation in the MAD. This correlation will only be observed in instances where relatively fast quasifission is a highly probable reaction outcome. In these cases, the MAD provides information on reaction time scales $[16,18]$.

In reactions where a mass-angle correlation is not observed, quasifission may still be an important reaction outcome. In these cases, angular anisotropies offer the clearest means of identifying reactions for which quasifission is important. This was seen in reactions involving the lightest three projectiles studied here. While none of these three reactions exhibited a mass-angle correlation, indicating a reaction timescale longer than a single rotation postcapture, all three exhibited enhanced angular anisotropies 
relative to transition state model calculations. The pattern of disagreement is likely to be due to nuclear structure effects, as discussed in Ref. [18]. In future work, improvements in the CCFULL inputs to these calculations along the lines of Ref. [31] may be useful for better identifying the appropriate Woods-Saxon potential parameters.

For all reactions, the widths of the mass ratio distributions only offered a clear signature of quasifission in cases where a mass-angle correlation was observed. In all other cases, mass distributions may provide some evidence of quasifission, but interpretation of this evidence requires model input.

To conclude, none of the signatures of quasifission presented here are capable of providing quantitative information on the importance of quasifission outcomes for a given reaction. However, the work presented here highlights the potential use of experimental MADs for the development of a dynamical model of fusion and fission. More importantly, it demonstrates that MADs are a sensitive probe of the type of reactions used in superheavy element studies; as such, such measurements offer a powerful tool for improving our understanding of the island of stability.

\section{Acknowledgments}

The authors acknowledge support from Australian Research Council Grants FL110100098 and DP110102858, and the Heavy Ion Accelerator Facility's accelerator and technical staff.

\section{References}

[1] B. Borderie, M. Berlanger, D. Gardès, F. Hanappe, L. Nowicki, J. Péter, B. Tamain, S. Agarwal, J. Girard, C. Grégoire et al., Zeit. Phys. A 299, 263 (1981)

[2] B.B. Back, R.R. Betts, K. Cassidy, B.G. Glagola, J.E. Gindler, L.E. Glendenin, B.D. Wilkins, Phys. Rev. Lett. 50, 818 (1983)

[3] B.B. Back, Phys. Rev. C 31, 2104 (1985)

[4] J. Tōke, B. Bock, G.X. Dai, A. Gobbi, S. Gralla, K.D. Hildenbrand, J. Kuzminski, W. Müller, A. Olmi, H. Stelzer, Nucl. Phys. A440, 327 (1985)

[5] D.J. Hinde, D. Hilscher, H. Rossner, B. Gebauer, M. Lehmann, M. Wilpert, Phys. Rev. C 45, 1229 (1992)

[6] D.J. Hinde, M. Dasgupta, J.R. Leigh, J.P. Lestone, J.C. Mein, C.R. Morton, J.O. Newton, H. Timmers, Phys. Rev. Lett. 74, 1295 (1995)

[7] D.J. Hinde, C.R. Morton, M. Dasgupta, J.R. Leigh, J.C. Mein, H. Timmers, Nucl. Phys. 592, 271 (1995)

[8] D.J. Hinde, M. Dasgupta, J.R. Leigh, J.C. Mein, C.R. Morton, J.O. Newton, H. Timmers, Phys. Rev. C 53, 1290 (1996)

[9] B.B. Back, P.B. Fernandez, B.G. Glagola, D. Henderson, S. Kaufman, J.G. Keller, S.J. Sanders, F. Videbæk, T.F. Wang, B.D. Wilkins, Phys. Rev. C 53, 1734 (1996)
[10] A.C. Berriman, D.J. Hinde, M. Dasgupta, C.R. Morton, R.D. Butt, J.O. Newton, Nature 413, 144 (2001)

[11] D.J. Hinde, M. Dasgupta, A. Mukherjee, Phys. Rev. Lett. 89, 282701 (2002)

[12] D.J. Hinde, R. du Rietz, M. Dasgupta, R.G. Thomas, L.R. Gasques, Phys. Rev. Lett. 101, 092701 (2008)

[13] R. Rafiei, R.G. Thomas, D.J. Hinde, M. Dasgupta, C.R. Morton, L.R. Gasques, M.L. Brown, M.D. Rodriguez, Phys. Rev. C 77, 024606 (2008)

[14] R.G. Thomas, D.J. Hinde, D. Duniec, F. Zenke, M. Dasgupta, M.L. Brown, M. Evers, L.R. Gasques, M.D. Rodriguez, A. Diaz-Torres, Phys. Rev. C 77, 034610 (2008)

[15] D.J. Kedziora, C. Simenel, Phys. Rev. C 81, 044613 (2010)

[16] R. du Rietz, D.J. Hinde, M. Dasgupta, R.G. Thomas, L.R. Gasques, M. Evers, N. Lobanov, A. Wakhle, Phys. Rev. Lett. 106, 052701 (2011)

[17] C. Simenel, D.J. Hinde, R. du Rietz, M. Dasgupta, M. Evers, C.J. Lin, D.H. Luong, A. Wakhle, Phys. Lett. B710, 607 (2012)

[18] E. Williams, D.J. Hinde, M. Dasgupta, R. du Rietz, I.P. Carter, M. Evers, D.H. Luong, S.D. McNeil, D.C. Rafferty, K. Ramachandran et al., Phys. Rev. C 88, 034611 (2013)

[19] R. du Rietz, E. Williams, D.J. Hinde, M. Dasgupta, M. Evers, C.J. Lin, D.H. Luong, C. Simenel, A. Wakhle, Phys. Rev. C 88, 054618 (2013)

[20] S. Mitsuoka, H. Ikezoe, K. Nishio, K. Satou, J. Lu, Phys. Rev. C 65, 054608 (2002)

[21] G.N. Knyazheva, E.M. Kozulin, R.N. Sagaidak, A.Y. Chizhov, M.G. Itkis, N.A. Kondratiev, V.M. Voskressensky, A.M. Stefanini, B.R. Behera, L. Corradi et al., Phys. Rev. C 75, 064602 (2007)

[22] B.B. Back, J. Phys.: Conf. Ser. 282, 012003 (2011)

[23] H.Q. Zhang, C.L. Zhang, C.J. Lin, Z.H. Liu, F. Yang, A.K. Nasirov, G. Mandaglio, M. Manganaro, G. Giardina, Phys. Rev. C 81, 034611 (2010)

[24] J.P. Lestone, J.R. Leigh, J.O. Newton, D.J. Hinde, J.X. Wei, J.X. Chen, S. Elfström, M. ZielinskaPfabé, Nucl. Phys. A559, 277 (1993)

[25] R. Vandenbosch, J.R. Huizenga, Nuclear Fission (Academic, New York, 1973)

[26] C.R. Morton, D.J. Hinde, J.R. Leigh, J.P. Lestone, M. Dasgupta, J.C. Mein, J.O. Newton, H. Timmers, Phys. Rev. C 52, 243 (1995)

[27] K. Hagino, N. Rowley, A.T. Kruppa, Comp. Phys. Comm. 129, 143 (1999)

[28] K. Hagino, N. Takigawa, Prog. of Theo. Phys. 128, 1061 (2012)

[29] J.C. Mein, D.J. Hinde, M. Dasgupta, J.R. Leigh, J.O. Newton, H. Timmers, Phys. Rev. C 55, R995 (1997)

[30] B.B. Back, R.R. Betts, J.E. Gindler, B.D. Wilkins, S. Saini, M.B. Tsang, C.K. Gelbke, W.G. Lynch, M.A. McMahan, P.A. Baisden, Phys. Rev. C 32, 195 (1985)

[31] C. Simenel, M. Dasgupta, D.J. Hinde, E. Williams, Phys. Rev. C 88, 064604 (2013) 